\title{
Computing the Geometric Mean Over Multiple- Access Channels: Error Analysis and Comparisons
}

\author{
Mario Goldenbaum and Sławomir Stańczak \\ Fraunhofer German-Sino Lab for Mobile Communications \\ Einsteinufer 37, D-10587 Berlin, Germany \\ Email: \{mario.goldenbaum, stanczak\}@ hhi.fraunhofer.de
}

\begin{abstract}
The paper deals with the problem of data transmission and function computation of the sensed data in wireless sensor networks, in which multiple sensor nodes transmit their data to one sink node over a wireless multiple-access channel. We focus on the problem of computing the geometric mean at the sink node by merging the data transmission and function computation into one step via an explicit utilization of channel collisions caused by simultaneous transmissions of sensor nodes. The paper provides the analysis of the estimation error and compares the scheme with traditional time division multiple-access based schemes to indicate potential for significant performance gains.
\end{abstract}

\section{INTRODUCTION}

In comparison to traditional wireless data networks which provide an end-to-end information transfer, wireless sensor networks are deployed to fulfill certain tasks in specific applications like environmental monitoring. Therefore, one is often not interested in reconstructing the sensed data from every individual (sensor) node, but rather in computing a function of the sensed data [1], [2]. Here, the objective is to compute some desired function of the sensed data, which can be for example arithmetic mean, geometric mean, maximum/minimum value. In order to compute a desired function, traditional schemes like time division multiple-access (TDMA) are usually used to avoid interference induced by concurrent transmissions of sensor nodes over a wireless channel with the broadcast property. In general, traditional approaches strictly separate the data transmission from the function computation, which is performed at some sensor nodes. Recently, however, it was shown in [3] that if the desired function "matches" the mathematical characteristic of the communication channel, the performance can be improved by merging the data transmission and function computation into one step, explicitly exploiting the channel collisions. This approach is known as Computation over Multiple-Access Channels (CoMAC) [3] and is a paradigm shift as the properties of the wireless channel are used to compute functions of sensor readings.

It is not surprising that the key element of the "natural" mathematical characteristic of a wireless multiple-access channel is simply summation (cf. Definition 1). However, if we allow certain pre-processing functions which operate on sensor data and certain post-processing functions which operate on the signal received by the sink node (cf. Definition 3 and 4),

This work was supported by the German Ministry for Education and Research (BMBF) under grant 01BU0680. it is also possible to compute nonlinear desired functions via the wireless multiple-access channel.

The original information theoretical concept by Nazer and Gastpar in [3] assumes perfect symbol- and phasesynchronization, which is illusive to ensure in a practical setup. Therefore, we proposed a simple scheme with high practical relevance [4], which only needs coarse block-synchronization. The analytical work in [4] was focused on the linear function "arithmetic mean". In this paper we extend the work of [4] to "geometric mean", which forms together with "arithmetic mean" important canonical representatives of the basic arithmetic operations "summation" and "multiplication". Thereby it turns out that geometric mean calculations over wireless multiple-access channels are much more difficult than arithmetic mean calculations due to the transformation of the "natural" mathematical characteristic (i.e., summation) of the wireless multiple-access channel to "multiplication".

The paper is organized as follows. In Section II we provide basic definitions and the principal idea behind CoMAC. In Section III we refine our previously proposed practical CoMAC approach, followed by a detailed error analysis of the desired function "geometric mean" in Section IV. Section V is devoted to numerical examples which indicate the accuracy of the theoretical analysis and to a comparison between CoMAC and an idealized TDMA scheme to visualize the remarkable performance gains. Finally, Section VI concludes the paper ${ }^{1}$.

\section{Definitions And Problem Statement}

Consider a wireless sensor network consisting of $K \in \mathbb{N}$ spatially distributed sensor nodes and one designated receiver node. Without loss of generality (w. l.o.g.) we assume that the $K$ nodes are identical, e.g., with respect to spectrum, data rate, bandwidth, symbol-duration, sensor elements.

\footnotetext{
${ }^{1}$ Notation: Random variables are denoted with uppercase letters and random vectors by bold uppercase letters, whereas corresponding realizations are denoted by lowercase equivalents. $\mathbb{N}, \mathbb{Z}_{+}, \mathbb{R}, \mathbb{R}_{+}, \mathbb{R}_{++}, \mathbb{C}$ denotes the sets of natural, nonnegative integer, real, nonnegative real, positive real, and complex numbers. The conjugate, transpose, and Hermitian transpose are denoted by $(\cdot)^{*},(\cdot)^{T}$ and $(\cdot)^{H} \cdot \mathcal{N}_{\mathbb{R}}\left(\mu, \sigma^{2}\right), \mathcal{N}_{\mathbb{C}}\left(\mu, \sigma^{2}\right)$ describe the real- and complex-valued normal distributions with mean $\mu$ and variance $\sigma^{2}, \mathcal{L} \mathcal{N}\left(\mu, \sigma^{2}\right)$ denotes the log-normal distribution, and $\chi_{n}^{2}$ the Chi-square distribution with $n$ degrees of freedom respectively. $\operatorname{Re}\{\cdot\}, \operatorname{Im}\{\cdot\}$ are the real and imaginary parts of a complex number, $\boldsymbol{I}_{n}$ is the $n \times n$ identity matrix, $\mathbf{1}_{n}$ the length $n$ column vector of all ones, and $\mathbb{1}_{\mathcal{A}}(x)$ the indicator function being equal to 1 if $x \in \mathcal{A}$ and 0 if $x \notin \mathcal{A}$. $\operatorname{erf}(\cdot), \operatorname{erfc}(\cdot)$ describe the error function and error function complement.
} 
Let an appropriate probability space $(\Omega, \mathcal{A}, \mathbb{P})$ be given, with sample space $\Omega, \sigma$-Algebra $\mathcal{A}$ and probability measure $\mathbb{P}$ over $\mathcal{A}$, over which all appearing random variables and stochastic processes are defined. Moreover, as a minor technicality ensuring that all random variables are well defined, we assume that occurring functions that are applied on random variables are Borel functions. The sensor nodes have the task to jointly observe a certain physical phenomenon and we model these observations as time-discrete stochastic processes $^{2} X_{k}: \Omega \times \mathbb{Z}_{+} \rightarrow \mathcal{X},(t, \omega) \mapsto X_{k}(t, \omega)$, $k=1, \ldots, K$. We assume that the joint probability density $p_{\boldsymbol{X}}(\boldsymbol{x} ; t):=p_{X_{1}, \ldots, X_{K}}\left(x_{1}, \ldots, x_{K} ; t\right)$ of sensor readings $\boldsymbol{X}(t):=\left(X_{1}(t), \ldots, X_{K}(t)\right)^{T} \in \mathcal{X}^{K}$ exists. The compact set $\mathcal{X}=\left[x_{\min }, x_{\max }\right] \subset \mathbb{R}$ denotes the physical measurement range, i.e., the range in which the measurement outcomes from physical phenomenon observations are.

In the following, we introduce the most important definitions for the analysis in this paper.

Definition 1 (WS-MAC): Let $W_{k}(\tau) \in \mathbb{R}, k=1, \ldots, K$, be a transmit symbol of node $k$ at time $\tau \in \mathbb{Z}_{+}$, which depends bijectively on the $k$ th measurement value $X_{k}(t)$ at time $t \in \mathbb{Z}_{+}$, and $P_{\max } \in \mathbb{R}_{++}$be the peak power constraint on each node. Let $H_{k}(\tau), k=1, \ldots, K$, be the complex-valued flat-fading process ${ }^{3}$ between the $k$ th sensor and the sink and let $N(\tau) \sim \mathcal{N}_{\mathbb{C}}\left(0, \sigma_{N}^{2}\right)$ be the white stationary time-discrete receiver noise process with stochastically independent real and imaginary parts, each with variance $\sigma_{N}^{2} / 2$. Assume that the data, the fading and the noise are mutually independent. Then, we refer to the map

$\left(W_{1}(\tau), \ldots, W_{K}(\tau)\right) \longmapsto \sum_{k=1}^{K} H_{k}(\tau) W_{k}(\tau)+N(\tau)=: Y(\tau)$,

$Y(\tau) \in \mathbb{C}$, as the Wireless Sensor Multiple-Access Channel (WS-MAC).

Definition 2 (Desired Function): $\mathcal{F}_{\mathrm{d}}$ is the set of desired functions $f: \mathcal{X}^{K} \rightarrow \mathbb{R}$ of measured sensor data given by

$$
f(\boldsymbol{X}(t))=(f \circ \boldsymbol{X})(t):=f\left(X_{1}(t), \ldots, X_{K}(t)\right) .
$$

Now, an important result of [3] was that if there is a match between the mathematical characteristic of the WSMAC and the desired function, the channel collisions induced by a simultaneous access of sensor nodes to the common air interface can be profitably used to merge the tasks of data transmission and function computation into one step. Equation (1) offers the natural mathematical characteristic of the WSMAC, which is obviously summation.

Definition 3 (Pre-processing Functions): We define the functions $\varphi_{k}: \mathcal{X} \rightarrow \mathbb{R}, k=1, \ldots, K$, which operate on the sensed data $X_{k}(t) \in \mathcal{X}$, i.e., $\varphi_{k}\left(X_{k}(t)\right)=\left(\varphi_{k} \circ X_{k}\right)(t)$, as the Pre-Processing Functions.

\footnotetext{
${ }^{2}$ Throughout the paper we skip the explicit designation of elementary events $\omega \in \Omega$ in the formulation of stochastic processes and write for example $X_{k}(t)$ instead of $X_{k}(t, \omega)$.

${ }^{3}$ Note that the transmission system is modeled in the complex baseband and w.l.o.g. path losses are incorporated in the fading gains $H_{k}(\tau) \forall k, \tau$.
}

Definition 4 (Post-Processing Function): Let $Y(t) \in \mathbb{C}$ be the output of the WS-MAC specified by Definition 1 . Then we define the injective function $\psi: \mathbb{R} \rightarrow \mathbb{R}$, which operate on $Y(t)$, i.e., $\psi(Y(t))=(\psi \circ Y)(t)$, as the Post-Processing Function.

Remark 1: The pre- and post-processing functions, which depend on the desired function, transform the WS-MAC such that the mathematical characteristic of the resulting overall channel matches the characteristic of the desired function. For example in the case of "geometric mean" as the desired function, the overall channel is a multiplicative multiple-access channel. Therefore, the set of desired functions $f$, which can be calculated by means of the WS-MAC (in a theoretical sense), has the form

$$
\mathcal{F}=\left\{f \in \mathcal{F}_{\mathrm{d}} \mid f(\boldsymbol{X}(t))=\psi\left(\sum_{k} \varphi_{k}\left(X_{k}(t)\right)\right)\right\},
$$

for some given sets of pre- and post-processing functions.

Example 1 (Desired Functions): (i) Arithmetic mean: $f(\boldsymbol{X}(t))=\frac{1}{K} \sum_{k=1}^{K} X_{k}(t)$ with pre-processing functions $\varphi_{k}\left(X_{k}(t)\right)=\varphi\left(X_{k}(t)\right)=X_{k}(t), k=1, \ldots, K$, and postprocessing function $\psi(Y(t))=\frac{1}{K} Y(t)$. (ii) Geometric mean: $f(\boldsymbol{X}(t))=\left(\prod_{k=1}^{K} X_{k}(t)\right)^{\frac{1}{K}}, X_{k}(t)>0 \forall k, t$, with preprocessing functions $\varphi_{k}\left(X_{k}(t)\right)=\varphi\left(X_{k}(t)\right)=\log _{a}\left(X_{k}(t)\right)$ $\forall k, t, \quad a$ an arbitrary base, and post-processing function $\psi(Y(t))=a^{\frac{1}{K} Y(t)}$. (iii) Weighted mean: $f(\boldsymbol{X}(t))=\frac{1}{\sum_{k=1}^{K} w_{k}} \sum_{k=1}^{K} w_{k} X_{k}(t), w_{k} \geq \overline{0 \forall k \text {, with pre- }}$ processing functions $\varphi_{k}\left(X_{k}(t)\right)=w_{k} X_{k}(t), k=1, \ldots, K$, and post-processing function $\psi(Y(t))=\frac{1}{\sum_{k=1}^{K} w_{k}} Y(t)$.

Now, the problem which arises is: How can we compute elements of (3) in an energy-efficient and robust way, by exploiting the broadcast property of the WS-MAC?

\section{Robust Analog Function Computation Over Wireless Sensor Multiple-ACCess Channels}

As mentioned in the introduction, the main drawback of the symbol-wise approach in [3] was the assumption of perfect symbol- and phase-synchronization, which is necessary to guarantee a constructive superposition of electro-magnetic waves in the sense of (1). However, as such an assumption is illusive in practical large-scale sensor networks, we proposed a simple analog scheme in [4], which desires only a coarse block-synchronization. In the following, we sum up and refine the idea of our previous work.

To compute a desired function using the WS-MAC, any sensor node generates a random unit norm transmit sequence $\boldsymbol{S}_{k}(t)=\left(S_{k 1}(t), \ldots, S_{k M}(t)\right)^{T} \in \mathbb{C}^{M}$ of length $M \in \mathbb{N}$ with elements

$$
S_{k m}(t)=\frac{1}{\sqrt{M}} \mathrm{e}^{i \Phi_{k m}(t)}, k=1, \ldots, K ; m=1, \ldots, M,
$$

where $i^{2}=-1$ and $\Phi_{k m}(t)$ uniformly and independent identically distributed (i.i.d.) in $[0,2 \pi) \forall k, m, t$.

Remark 2: Due to implementation issues it is not necessary that the phases $\Phi_{k m}(t)$ are continuous random variables for any time instance as formulated in (4). Without performance 


$$
\begin{aligned}
\boldsymbol{Y}(t)^{H} \boldsymbol{Y}(t)= & \alpha \sum_{k=1}^{K}\left|H_{k}(t)\right|^{2} \varphi_{k}\left(R_{k}(t)\right)+\alpha \underbrace{\sum_{k=1}^{K} \sum_{\ell=1}^{K} H_{k}(t)^{*} H_{\ell}(t) \sqrt{\varphi_{k}\left(R_{k}(t)\right) \varphi_{\ell}\left(R_{\ell}(t)\right)} \boldsymbol{S}_{k}(t)^{H} \boldsymbol{S}_{\ell}(t)}_{=: \Delta_{1}(t)} \\
& +2 \sqrt{\alpha} \underbrace{\sum_{k=1}^{K} \sqrt{\varphi_{k}\left(R_{k}(t)\right)} \operatorname{Re}\left\{H_{k}(t)^{*} \boldsymbol{S}_{k}(t)^{H} \boldsymbol{N}(t)\right.}_{=: \Delta_{2}(t)}\}+\underbrace{\boldsymbol{N}(t)^{H} \boldsymbol{N}(t)}_{=: \widetilde{N}(t)}
\end{aligned}
$$

loss it is sufficient that the $\Phi_{k m}(t)$ attain a finite number $L=$ $2^{m}, m \in \mathbb{N}, m \geq 2$, of values

$$
\Phi_{k m}(t) \equiv 2 \pi L^{-1} \Upsilon_{k m}(t) \quad \forall k, m, t,
$$

with $\Upsilon_{k m}(t)$ uniformly and i.i.d. distributed over $\{1, \ldots, L\}$ $\forall k, m, t$, i.e., $\Upsilon_{k m}(t)=\left\lceil L \cdot \Gamma_{k m}(t)\right\rceil$, where $\Gamma_{k, m}(t)$ uniformly and i.i.d. distributed over $[0,1] \forall k, m, t$, which simplifies the sequence generation on nodes.

The energy of each transmit sequence, say sequence $\boldsymbol{S}_{k}(t)$, is set to be equal to the pre-processed sensor reading $\varphi_{k}\left(X_{k}(t)\right)$, which reveals that we have to satisfy $\varphi_{k}\left(X_{k}(t)\right) \geq$ $0 \forall k, t$, because transmit energies are nonnegative real numbers. Therefore, we map the physical measurement range $\mathcal{X}$ bijectively onto the measurement range, defined as follows.

Definition 5 (Measurement Range): Let $\mathcal{X}$ be the physical measurement range of the sensors and $g_{\varphi}: \mathcal{X} \rightarrow \mathcal{R}$ a pre-processing dependent bijective function, such that for all $X_{k}(t) \in \mathcal{X}, t \in \mathbb{Z}_{+}, R_{k}(t):=g_{\varphi}\left(X_{k}(t)\right)=\left(g_{\varphi} \circ X_{k}\right)(t) \in$ $\mathcal{R}(k=1, \ldots, K)$. Then we refer to the interval $\mathcal{R}=$ $\left[r_{\min }, r_{\max }\right] \subset \mathbb{R}$ as the Measurement Range of sensor nodes if and only if $\varphi_{k}\left(R_{k}(t)\right) \geq 0 \forall R_{k}(t) \in \mathcal{R}, k=1, \ldots, K$, $t \in \mathbb{Z}_{+}$.

Example 2 (Measurement Ranges): (i) Arithmetic mean: $g_{\varphi}\left(X_{k}(t)\right)=R_{k}(t)=X_{k}(t)+\left|x_{\min }-\varepsilon\right|, \varepsilon>0$ appropriately chosen, if $x_{\min } \leq 0$ and $g_{\varphi}\left(X_{k}(t)\right)=X_{k}(t)$ if $x_{\min }>0$. For the measurement range follows $\mathcal{R}=\left[\varepsilon, x_{\max }+\left|x_{\min }-\varepsilon\right|\right]$ for $x_{\min } \leq 0$ and $\mathcal{R}=\mathcal{X}$ for $x_{\min }>0$. (ii) Geometric mean: Let $X_{k}(t) \in \mathcal{X}=\left[x_{\min }, x_{\max }\right]$ with $0<x_{\min }<x_{\max } \forall k, t$, $g_{\varphi}\left(X_{k}(t)\right)=R_{k}(t)=b X_{k}(t)$, with $b=\frac{1}{x_{\min }-\varepsilon}, \varepsilon>0$ appropriately chosen, so that the measurement range is $\mathcal{R}=\left[\frac{x_{\min }}{x_{\min }-\varepsilon}, \frac{x_{\max }}{x_{\min }-\varepsilon}\right]$ if $x_{\min } \leq 1$ and $\mathcal{R}=\mathcal{X}$ otherwise.

Remark 3: The parameter $\varepsilon$ in Example 2 ensures the strict positivity of transmit energies. From a theoretical point of view this is not necessary, but in practice it allows to distinguish between the special case $f(\boldsymbol{x}(t))$, with $\boldsymbol{x}(t)=x_{\min } \mathbf{1}_{K}$, and a malfunctioning computation network.

With the considerations above, the sampled complex vectorvalued receive process at the output of the WS-MAC has the explicit form

$$
\boldsymbol{Y}(t)=\sum_{k=1}^{K} H_{k}(t) \sqrt{\alpha \varphi_{k}\left(R_{k}(t)\right)} \boldsymbol{S}_{k}(t)+\boldsymbol{N}(t)
$$

with $\boldsymbol{N}(t)=\left(N_{1}(t), \ldots, N_{M}(t)\right)^{T} \in \mathbb{C}^{M}$ being vectorvalued additive Gaussian noise, i.e., $\boldsymbol{N}(t) \sim \mathcal{N}_{\mathbb{C}}\left(\mathbf{0}, \frac{1}{M} \sigma_{N}^{2} \boldsymbol{I}_{M}\right)$ with $\operatorname{Re}\{\boldsymbol{N}(t)\}, \operatorname{Im}\{\boldsymbol{N}(t)\} \sim \mathcal{N}_{\mathbb{R}}\left(\mathbf{0}, \frac{1}{2 M} \sigma_{N}^{2} \boldsymbol{I}_{M}\right)$ mutually independent ${ }^{4}$ for all $t \in \mathbb{Z}_{+}, H_{k}(t) \in \mathbb{C}$ the frequency-flat block-fading process of sensor $k$, and $\alpha \in \mathbb{R}_{++}$a constant being chosen to satisfy transmit power constraints on sensor nodes, i.e., $0 \leq \alpha \varphi_{k}\left(R_{k}(t)\right) / M \leq P_{\max } \forall R_{k}(t), k=$ $1, \ldots, K$. If the pre-processing functions are monotonically increasing and identical for $\forall k$, then $\alpha=\frac{M P_{\max }}{\varphi\left(r_{\max }\right)}$, whereas $\alpha=\frac{M P_{\max }}{\max _{k}\left\{\max _{r \in \mathcal{R}}\left\{\varphi_{k}(r)\right\}\right\}}$ if the pre-processing functions are different on nodes and $\alpha=\frac{M P_{\max }}{\max _{k}\left\{\varphi_{k}\left(r_{\max }\right)\right\}}$ if the preprocessing functions are monotonically increasing but different $\forall k$ respectively, as for example in the case of desired function "weighted mean" (cf.Example 1 (iii)).

Due to the fact that the described scheme requires an adaptation of sensor readings to yield valid transmit energies after the application of pre-processing functions, the subset of desired functions (3) has now changed to

$$
\begin{array}{r}
\widetilde{\mathcal{F}}=\left\{f \mid f(\boldsymbol{X}(t))=g_{\varphi}^{-1}\left(\psi\left(\sum_{k} \varphi_{k}\left(g_{\varphi}\left(X_{k}(t)\right)\right)\right)\right),\right. \\
\left.\varphi_{k} \geq 0 \forall g_{\varphi}\left(X_{k}(t)\right), k=1, \ldots, K\right\} \subset \mathcal{F}_{\mathrm{d}},
\end{array}
$$

where $\psi, \varphi_{k}$ are taken from some given sets of pre- and postprocessing functions and $g_{\varphi}$ an appropriate function according to Definition 5. We have to mention that the cardinality of $\widetilde{\mathcal{F}}$ reduces in practice due to restrictions as for example power constraints

To reconstruct/estimate the desired function from the received vector (5), the sink node first has to compute the received energy to obtain (7) at the top of the page, followed by simple calculations to yield a function estimate with certain properties (cf. Section IV-B). Besides the noise energy summand $\widetilde{N}(t) \in \mathbb{R}_{+}$, two additional error terms $\Delta_{1} \in \mathbb{R}$ and $\Delta_{2} \in \mathbb{R}$ appear, which encompass cross-correlations between the transmit sequences itself and between transmit sequences and noise. Furthermore the first term in (7) shows the desired term and offers that the sensor nodes require instantaneous knowledge about the complex-valued channel gains $H_{k}(t)$ prior to transmissions. In this paper we assume that the sink node initiates a function value transmission through pilot sequences, such that the sensor nodes are able to

\footnotetext{
${ }^{4}$ The scaling with $\frac{1}{\sqrt{M}}$ in (4) induces a constant energy per function value transmission (independent of $M$ ), which is the reason why the covariance matrix $\frac{1}{M} \sigma_{N}^{2} \boldsymbol{I}_{M}$ of the complex noise vector $\boldsymbol{N}(t)$ scales also with $\frac{1}{M}$ to ensure a constant noise energy per function value transmission.
} 
estimate their own channel coefficients and invert the channels perfectly. With this assumption, (7) at the top of the page can be compactly written as

$$
\boldsymbol{Y}(t)^{H} \boldsymbol{Y}(t)=\alpha \sum_{k=1}^{K} \varphi_{k}\left(R_{k}(t)\right)+\widetilde{\Delta}(t),
$$

where

$$
\widetilde{\Delta}(t):=\alpha \Delta_{1}(t)+2 \sqrt{\alpha} \Delta_{2}(t)+\widetilde{N}(t)
$$

constitutes the sum of error terms.

The main advantage of the analog scheme described above in comparison to traditional schemes is the fact that no sensitive symbol- and phase-synchronization is necessary. Only a coarse block-synchronization is required to ensure that the transmit sequences reach the sink node with a significant overlap. Therefore, no explicit protocol structure which induces a noticeable amount of overhead is necessary, such that computation networks following this design rule are energy- and complexity-efficient and can be easily implemented in practice. Finally, the hardware-effort is reduced because energy consuming digital components as analog-to-digital converters, registers, etc. are no longer required.

\section{Geometric Mean Analysis}

In this section we analyze the computation performance of the scheme described in Section III with respect to the desired function "geometric mean". Geometric mean calculations in wireless sensor networks are only appropriate for applications where sensed values are greater than zero, i.e., $X_{k}(t)>0$ $\forall k, t$ or rather $X_{k}(t) \in \mathcal{X}=\left[x_{\text {min }}, x_{\text {max }}\right]$ with $0<x_{\text {min }}<$ $x_{\text {max }} \forall k, t$ (cf. Example 2 (ii)). For simplicity, we focus in the following on an arbitrary but fixed time instance $t \in \mathbb{Z}_{+}$and drop a corresponding designation.

Definition 6 (Function Recovery Error): Let $f(\boldsymbol{X}), \boldsymbol{X} \in$ $\mathcal{X}^{K}$, be the desired function and $\hat{f}(\boldsymbol{X})$ a corresponding estimate at the sink node. Then we define

$$
E:=(\hat{f}(\boldsymbol{X})-f(\boldsymbol{X})) / f(\boldsymbol{X}), f(\boldsymbol{X}) \neq 0 \forall \boldsymbol{X} \in \mathcal{X}^{K}
$$

as the relative Function Recovery Error.

Similar to [4], we will measure the computation performance at the sink node by means of the probability that the absolute value of the relative function recovery error is greater or equal to $\epsilon>0$, i.e., $\mathbb{P}(|E| \geq \epsilon)$.

\section{A. Approximated Error Distribution}

To analyze the geometric mean performance according to Definition 6, we must first define an appropriate function value estimator based on the received energy (8).

Definition 7 (Heuristic Function Estimate): Let $f$ be the desired function "geometric mean". Then, we define the heuristic function estimate as

$$
\hat{f}(\boldsymbol{X}):=\frac{g_{\varphi}^{-1}\left(\psi\left(\frac{1}{\alpha} \boldsymbol{Y}^{H} \boldsymbol{Y}\right)\right)}{\mathbb{E}\left\{\psi\left(\frac{1}{\alpha} \widetilde{N}\right)\right\}}=f(\boldsymbol{X}) \frac{\psi\left(\frac{1}{\alpha} \widetilde{\Delta}\right)}{\mathbb{E}\left\{\psi\left(\frac{1}{\alpha} \widetilde{N}\right)\right\}}
$$

with $\mathbb{E}\left\{\psi\left(\frac{1}{\alpha} \tilde{N}\right)\right\} \geq 1$ and $\varphi, \psi, g_{\varphi}$ as defined in Example 1 (ii) and 2 (ii) respectively.
Remark 4: Note that in general, any individual desired function needs its own adequate estimator. In particular, the heuristic geometric mean estimator in Definition 7 is not unbiased. The unbiased formulation

$$
\hat{f}(\boldsymbol{X})=f(\boldsymbol{X}) \psi\left(\frac{1}{\alpha} \widetilde{\Delta}\right) \mathbb{E}\left\{\psi\left(\frac{1}{\alpha} \widetilde{\Delta}\right)\right\}^{-1}
$$

from [4] is however not applicable in practice, because the denominator depends on the distribution of sensor readings, which is usually unknown at the sink. As mentioned before, in the case of "geometric mean" the WS-MAC is transformed into a multiplicative multiple-access channel, why the estimation deviation has also a multiplicative nature.

An implementation of the heuristic estimator (10) in practice requires the knowledge of the expected value $\mathbb{E}\left\{\psi\left(\frac{1}{\alpha} \widetilde{N}\right)\right\}$ at the sink, which is explicitly given in part (i) of the following lemma. Part (ii) will be used in Section IV-B.

Lemma 1: Let $\widetilde{N}$ be defined as in (7), $a>1$ an arbitrary base commonly used by the sensor nodes, $\alpha>0$, and $\sigma_{N}^{2} \log _{\mathrm{e}}(a)<\alpha K M$. Then

(i) $\mathbb{E}\left\{\psi\left(\frac{1}{\alpha} \tilde{N}\right)\right\}=\mathbb{E}\left\{a^{\frac{1}{\alpha K} \widetilde{N}}\right\}=\left(\frac{\alpha K M}{\alpha K M-\sigma_{N}^{2} \log _{\mathrm{e}}(a)}\right)^{M}$

(ii) $\lim _{M \rightarrow \infty} \mathbb{E}\left\{\psi\left(\frac{1}{\alpha} \tilde{N}\right)\right\}=\mathrm{e}^{\frac{\sigma_{N}^{2} \log _{\mathrm{e}}(a)}{\alpha K}}$.

Proof: The proof is deferred to Appendix A.

Remark 5: Note that the expected value in Lemma 1 (i) does not exist for $\sigma_{N}^{2} \log _{\mathrm{e}}(a) \geq \alpha K M$, but the opposite is fulfilled in almost any practical situation.

With the heuristic estimator from Definition 7, the relative function recovery error in the context of geometric mean calculations has the explicit form

$$
E=\frac{\psi\left(\frac{1}{\alpha} \widetilde{\Delta}\right)}{\mathbb{E}\left\{\psi\left(\frac{1}{\alpha} \widetilde{N}\right)\right\}}-1=\frac{a^{\frac{1}{\alpha K} \widetilde{\Delta}}}{\mathbb{E}\left\{a^{\frac{1}{\alpha K} \widetilde{N}}\right\}}-1=\frac{1}{\gamma} \Xi-1,
$$

with $\gamma:=\mathbb{E}\left\{\psi\left(\frac{1}{\alpha} \widetilde{N}\right)\right\}$ and $\Xi:=\psi\left(\frac{1}{\alpha} \widetilde{\Delta}\right)$.

To evaluate $\mathbb{P}(|E| \geq \epsilon), \epsilon>0$, we need obviously an expression for the distribution function of $|E|=\mid \gamma^{-1} \Xi-$ $1 \mid$. To this end, we first calculate the distribution function of $\Xi$ conditioned on an arbitrary realization $\boldsymbol{x}=$ $\left(g_{\varphi}^{-1}\left(r_{1}\right), \ldots, g_{\varphi}^{-1}\left(r_{K}\right)\right)^{T}$ of sensor readings. Unfortunately, the exact determination of the distribution of $\Xi \mid x$ is difficult. However, we derive an approximated distribution in the following lemma.

Lemma 2: Let $\Xi \mid \boldsymbol{x}=\psi\left(\frac{1}{\alpha} \widetilde{\Delta} \mid \boldsymbol{x}\right)=a^{\frac{1}{\alpha K} \widetilde{\Delta} \mid \boldsymbol{x}}$ as defined in (12) with $\widetilde{\Delta}$ the error-sum in (9), both conditioned on a realization of sensor readings. Then, for arbitrary but fixed $K<\infty$, compact $\mathcal{X}$, and $M$ sufficiently large, $\Xi \mid \boldsymbol{x}$ is approximately $\mathcal{L} \mathcal{N}\left(\mu_{\Xi}, \sigma_{\Xi \mid \boldsymbol{x}}^{2}\right)$ distributed with parameters $\mu_{\Xi}=\sigma_{N}^{2} \log _{\mathrm{e}}(a) /(\alpha K)$ and $\sigma_{\Xi \mid \boldsymbol{x}}^{2}=\sigma_{\breve{\Delta} \mid \boldsymbol{x}}^{2}\left(\log _{\mathrm{e}}(a)\right)^{2} / K^{2}$, where $\sigma_{\breve{\Delta} \mid \boldsymbol{x}}^{2}$ denotes the variance of $\breve{\Delta} \mid \boldsymbol{x}:=\frac{1}{\alpha}\left(\widetilde{\Delta} \mid \boldsymbol{x}-\sigma_{N}^{2}\right)$, which was explicitly calculated in [4].

Proof: The proof is deferred to Appendix B.

With Lemma 2 in hand we are able now to state the main result of the current subsection.

Proposition 1: Let $E$ be the relative function recovery error as in Definition 6, $\mu_{\Xi}, \sigma_{\Xi \mid \boldsymbol{x}}^{2}$ as defined in Lemma 2, and $\gamma$ as 
defined in (12) (explicitly given in Lemma 1 (i)). Then, for $M$ large enough, the error probability $\mathbb{P}(|E| \geq \epsilon), \epsilon>0$, of the desired function "geometric mean" can be approximated by

$$
\begin{aligned}
\mathbb{P}(|E| \geq \epsilon) & =\int_{\mathcal{X}^{K}} \mathbb{P}(|E| \geq \epsilon \mid \boldsymbol{X}=\boldsymbol{x}) p_{\boldsymbol{X}}(\boldsymbol{x}) d \boldsymbol{x} \\
& \approx \int_{\mathcal{X}^{K}} \widetilde{\mathbb{P}}(|E| \geq \epsilon \mid \boldsymbol{X}=\boldsymbol{x}) p_{\boldsymbol{X}}(\boldsymbol{x}) d \boldsymbol{x}
\end{aligned}
$$

with

$$
\begin{aligned}
& \mathbb{P}(|E| \geq \epsilon \mid \boldsymbol{X}=\boldsymbol{x}) \approx \widetilde{\mathbb{P}}(|E| \geq \epsilon \mid \boldsymbol{X}=\boldsymbol{x})= \\
& \left\{\begin{array}{lc}
\frac{1}{2}\left[2+\operatorname{erf}\left(\frac{\log _{\mathrm{e}}(\gamma-\gamma \epsilon)-\mu_{\Xi}}{\sqrt{2 \sigma_{\Xi \mid x}^{2}}}\right)-\operatorname{erf}\left(\frac{\log _{\mathrm{e}}(\gamma+\gamma \epsilon)-\mu_{\Xi}}{\sqrt{2 \sigma_{\Xi \mid \boldsymbol{x}}^{2}}}\right)\right], \\
0<\epsilon<1 \\
\frac{1}{2} \operatorname{erfc}\left(\frac{\log _{\mathrm{e}}(\gamma+\gamma \epsilon)-\mu_{\Xi}}{\sqrt{2 \sigma_{\Xi \mid \boldsymbol{x}}^{2}}}\right), & 1 \leq \epsilon<\infty .
\end{array}\right.
\end{aligned}
$$

Proof: The proof is deferred to Appendix C.

In Section V-A we choose a particular density $p_{\boldsymbol{X}}(\boldsymbol{x})$ and evaluate (13) numerically to indicate the accuracy of the approximation for different network parameters.

\section{B. Asymptotic Estimation Properties}

On the basis of Proposition 1 we now conclude important properties of the heuristically motivated estimator from Definition 7.

Proposition 2: Let $K<\infty$ arbitrary but fixed and $\mathcal{X} \subset \mathbb{R}$ be compact. Then, with respect to the sequence length $M$, the heuristic estimator $\hat{f}$ from Definition 7 is consistent, i.e., $\lim _{M \rightarrow \infty} \mathbb{P}(|\hat{f}(\boldsymbol{X})-f(\boldsymbol{X})| \geq \epsilon)=0 \forall \epsilon>0$.

Proof: Due to the lack of space we skip the proof, but note as a sketch that for the proof it is sufficient to show for all $\epsilon>0$ the uniform convergence of (14) on $\mathcal{X}^{K}$ to the zero function as $M$ tends to infinity.

Remark 6: Of course Proposition 2 implies that (10) is also asymptotically unbiased, i.e., $\lim _{M \rightarrow \infty} \mathbb{E}\{\hat{f}(\boldsymbol{X}) \mid \boldsymbol{X}=\boldsymbol{x}\}=$ $f(\boldsymbol{x}) \forall \boldsymbol{x} \in \mathcal{X}^{K}$, which can be directly verified using Lemma 1 (ii) and the well known shape of the expectation value of a $\mathcal{L} \mathcal{N}\left(\mu_{\Xi}, \sigma_{\Xi, \boldsymbol{x}}^{2}\right)$ distributed random variable. Therewith the heuristic estimator (10) and the impractical estimator (11) are asymptotically equivalent.

\section{NUMERICAL EXAMPLES}

Without loss of generality, we consider in this section an explicit environmental monitoring scenario in which the sensors observe temperatures and a designated sink node is interested in the "geometric mean" of sensor readings. The aim of the numerical examples is to indicate that our theoretical analysis and approximations are quite accurate, that the heuristic estimator from Definition 7 performs very well, and finally that the analog function computation approach from Section III outperforms TDMA-like schemes in typical wireless sensor network operating points.

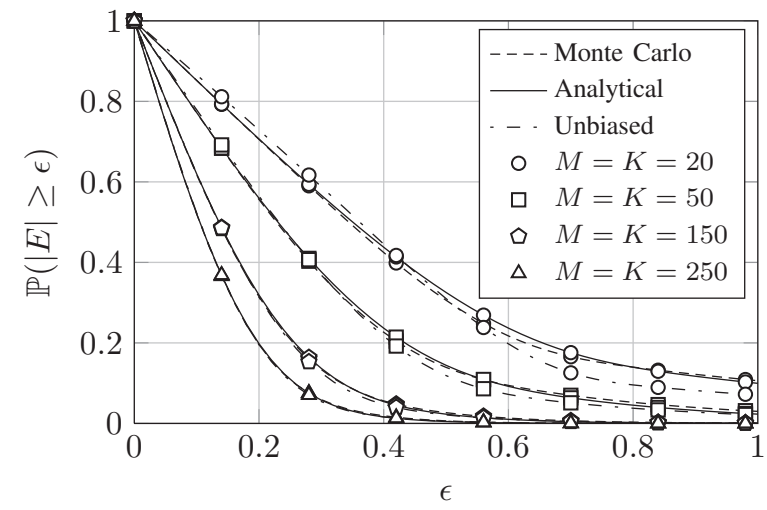

Fig. 1. Monte Carlo simulations $\left(10 \cdot 10^{3}\right.$ realizations) vs. analytical results with the heuristic estimator (10) and the impractical unbiased estimator (11), for different $M$ and $K=M$.

\section{A. Accuracy of Theoretical Analysis}

In this subsection we compare the results (13), (14) using the heuristic estimator (10) with Monte Carlo evaluations of $\mathbb{P}(|E| \geq \epsilon)$ and Monte Carlo evaluations using the unbiased estimator (11) for different network sizes/sequence lengths.

Example 3 (Accuracy): Let $K=20,50,150,250$, the sequence length $M=K, \sigma_{N}^{2}=1, a=2$, and the sensor readings uniformly and i.i.d. in $\mathcal{X}=\left[0.5^{\circ} \mathrm{C}, 14^{\circ} \mathrm{C}\right]$. The resulting experimental data are depict in Fig. 1.

The plots indicate that our analytical results are quite accurate, also for short sequence lengths. The insignificant difference for $M=20$ between our analytical results and the Monte Carlo simulations results from the fact that $\Xi \mid \boldsymbol{x}$ in (12) is only approximately log-normal distributed (cf. Lemma 2) and the relatively small number of summands in (9) tends to a poorer approximation accuracy. But the difference vanishes quickly with growing $M$.

The discrepancy for short sequence lengths between the heuristic estimator and (11) can be neglected, because the difference appears in a $\epsilon$-range which is not of much interest. Furthermore, the plots confirm that the heuristic estimator is asymptotically unbiased with a quick convergence.

\section{B. Comparisons With TDMA}

Now we compare the CoMAC approach from Section III with an idealized uncoded low complexity BPSK modulated TDMA scheme, which performs a hard decision at the sink node followed by a uniform quantization of sensor readings with $Q \in \mathbb{N}$ bit over the sensing range defined as follows.

Definition 8 (Sensing Range): The Sensing Range of sensor elements, i.e., the hardware dependent range in which the sensor elements can quantify values, is defined by the compact interval $\mathcal{S}=\left[s_{\min }, s_{\max }\right]$.

Obviously, $\mathcal{X} \subseteq \mathcal{S}$ should be fulfilled for adequate environmental monitoring.

Since the uniform quantization for TDMA is performed over $\mathcal{S}$, the sensing range will be partitioned into $2^{Q}$ nonoverlapping regions such that each sensor separately has to transmit a bit stream of length $Q$ to the sink. 
To ensure fairness between CoMAC and TDMA, with fixed degrees of freedom (bandwidth, symbol duration), both schemes should induce the same costs per function value transmission with respect to "transmit energy" and "transmit time". More precisely, this means that $\mathrm{E}_{\mathrm{CoMAC}, k}=\mathrm{E}_{\mathrm{TDMA}, k}$, $k=1, \ldots, K$, and $T_{\mathrm{CoMAC}}=T_{\mathrm{TDMA}}$ has to be fulfilled, with $\mathrm{E}_{\mathrm{CoMAC}, k} \in \mathbb{R}_{+}$the instantaneous CoMAC transmit energy of node $k, \mathrm{E}_{\mathrm{TDMA}, k} \in \mathbb{R}_{+}$the corresponding instantaneous TDMA transmit energy, $T_{\text {CoMAC }} \in \mathbb{R}_{++}$the transmission time per function value for CoMAC and $T_{\mathrm{TDMA}} \in \mathbb{R}_{++}$the transmission time per function value for TDMA respectively. Let $T \in \mathbb{R}_{++}$be the common symbol duration, $P_{\text {CoMAC, } k}=$ $\frac{\alpha \varphi\left(R_{k}(t)\right)}{M} \in \mathbb{R}_{+}$the instantaneous CoMAC transmit power of sensor $k$ and $P_{\text {TDMA, } k} \in \mathbb{R}_{+}$the instantaneous TDMA equivalent. Then, the transmit times per function value are $T_{\mathrm{CoMAC}}=M T$ and $T_{\mathrm{TDMA}}=Q K T$, whereas the transmit energies can be now formulated as $\mathrm{E}_{\mathrm{CoMAC}, k}=M P_{\mathrm{CoMAC}, k} T$ and $\mathrm{E}_{\mathrm{TDMA}, k}=Q P_{\mathrm{TDMA}, k} T$, respectively. Hence, to guarantee fairness in the sense described above, for the CoMAC sequence length it follows immediately that $M=Q K$ and the required instantaneous TDMA transmit power can be calculated as $P_{\mathrm{TDMA}, k}=\frac{P_{\mathrm{CoMAC}, k} M}{Q}=\frac{\alpha \varphi_{k}\left(R_{k}(t)\right)}{Q}, k=1, \ldots, K$.

Besides the fairness aspects, an adequate scheme comparison also requires the determination of a system operating point, which can be done in terms of a signal-to-noise ratio (SNR). Since the transmit energies are random variables in our scheme, it is appropriate to consider averaged signal-to-noise ratios. For simplicity, we assume the sensed values $X_{k}(t)$ are i.i.d. in $\mathcal{X} \forall k, t$, such that for the desired function "geometric mean" the averaged received TDMA-SNR per node (averaged over sensor readings) can be defined as

$$
\overline{\mathrm{SNR}}_{\mathrm{geo}}:=\frac{\bar{P}_{\mathrm{TDMA}, 1}}{\frac{\sigma_{N}^{2}}{2 M}}=\frac{\alpha \mathbb{E}\left\{\log _{a}\left(b X_{1}(t)\right)\right\}}{Q \cdot \frac{\sigma_{N}^{2}}{2 M}},
$$

with $\alpha=\frac{M P_{\max }}{\log _{a}\left(b x_{\max }\right)}$ and $\mathbb{E}\left\{\log _{a}\left(b X_{1}(t)\right)\right\}$ calculated as

$$
\frac{1}{\log _{\mathrm{e}}(a)}\left(\frac{x_{\max } \log _{\mathrm{e}}\left(b x_{\max }\right)-x_{\min } \log _{\mathrm{e}}\left(b x_{\min }\right)}{x_{\max }-x_{\min }}-1\right) \text {. }
$$

If we allow a certain $\overline{\mathrm{SNR}}_{\text {geo }}$ in $\mathrm{dB}$, i.e., $\overline{\mathrm{SNR}}_{\text {geo }}^{\mathrm{dB}}=$ $10 \log _{10}\left(\overline{\mathrm{SNR}}_{\mathrm{geo}}\right)$, the corresponding system operating point can be determined through an appropriate choice of $P_{\max }$ for fixed $\sigma_{N}^{2}$, or alternatively by choosing

$$
\sigma_{N}^{2}=\alpha 2 M Q^{-1} \mathbb{E}\left\{\log _{a}\left(b X_{1}(t)\right)\right\} 10^{-\frac{\overline{\mathrm{SNR}}_{\mathrm{ge}}^{\mathrm{dB}}}{10}},
$$

$P_{\max }$ fixed, for TDMA and CoMAC, respectively. In the following we prefer the second approach by adjusting $\sigma_{N}^{2}$ instead of $P_{\max }$ w. 1. o. g.

For the comparison examples below, let the physical measurement range be $\mathcal{X}=\left[0.5^{\circ} \mathrm{C}, 14^{\circ} \mathrm{C}\right]$ and $X_{k}(t)$ be i.i.d. uniformly distributed in $\mathcal{X} \forall k, t$. For the measurement range it follows immediately that $\mathcal{R}=\left[\frac{x_{\min }}{x_{\min }-\varepsilon}, \frac{x_{\max }}{x_{\min }-\varepsilon}\right]=\left[\frac{1}{1-2 \varepsilon}, \frac{28}{1-2 \varepsilon}\right]$ (cf.Example 2(ii)) and w. l.o.g. we set $\varepsilon=10^{-2}$. Furthermore, let the sensing range be $\mathcal{S}=\left[-55^{\circ} \mathrm{C}, 130^{\circ} \mathrm{C}\right]$, which is typical for ultra-low power temperature sensors [5].

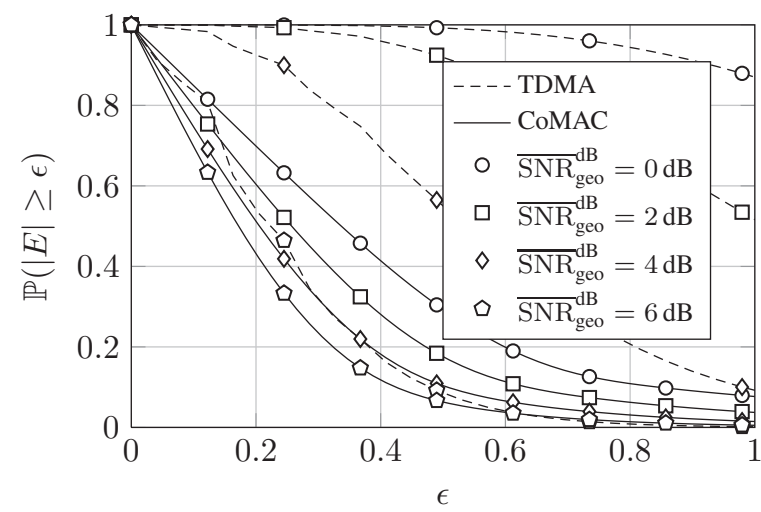

Fig. 2. CoMAC vs. uncoded TDMA for $K=25$ sensor nodes, quantization with $Q=8$ bit, sequence length $M=Q K$, and different averaged SNRs.

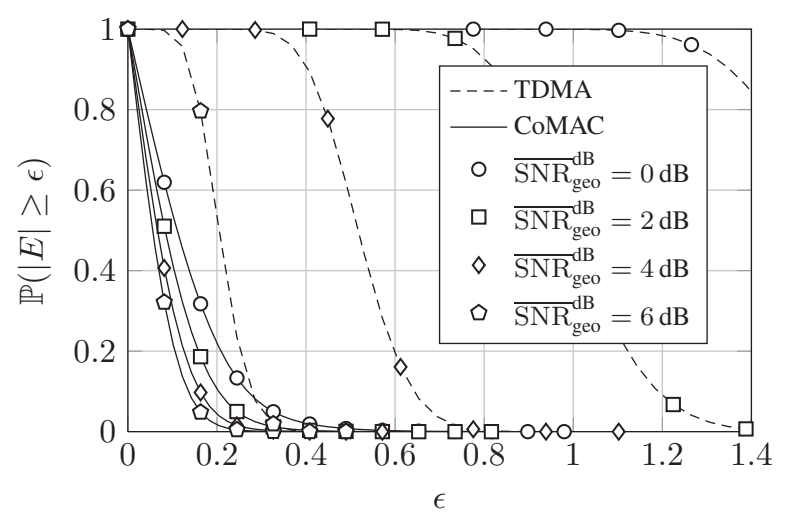

Fig. 3. CoMAC vs. uncoded TDMA for $K=250$ sensor nodes, quantization with $Q=8$ bit, sequence length $M=Q K$, and different averaged SNRs.

Example 4 (Small Network Size): Let $K=25, Q=8$ bit, the sequence length $M=Q K, \overline{\mathrm{SNR}}_{\text {geo }}^{\mathrm{dB}}=0,2,4,6 \mathrm{~dB}$ (low power regime), $P_{\max }=100 \mathrm{~mW}$, and $\sigma_{N}^{2}$ chosen according to (17). The corresponding simulation data is depicted in Fig. 2.

Example 5 (Medium Network Size): Let $K=250$ and all other simulation parameters as in Example 4. The corresponding simulation data is shown in Fig. 3.

The comparisons indicate the potential of the practical scheme described in Section III for nonlinear function computations. In particular, in the sensor network typical low power regime, CoMAC entirely outperforms TDMA with respect to the function recovery quality. This is also true for small network sizes, as shown in Fig. 2.

Note that the performance gains are quite conservative, since the simulated TDMA scheme was idealized in many ways. For example, a practical TDMA would require an established protocol stack with considerable amount of overhead such that the overall TDMA transmission time extends significantly.

\section{CONCLUSION}

In this contribution we refined the analog function computation approach recently proposed in [4] and demonstrated that it is able to efficiently compute nonlinear desired functions such the "geometric mean". In order to achieve this, it was 
necessary to formulate a heuristic function estimator and analyze its asymptotic properties, because the previously proposed unbiased one is not applicable in practice. Subsequently, the function recovery performance was analytically examined and also validated by numerical examples. Finally, we numerically compared the approach with an idealized TDMA scheme for different system parameters, indicating that CoMAC entirely outperforms TDMA for typical averaged SNRs.

\section{APPENDIX A \\ PROOF OF LEMMA 1}

Proof: (i) Since $\boldsymbol{N} \sim \mathcal{N}_{\mathbb{C}}\left(\mathbf{0}, \frac{\sigma_{N}^{2}}{M} \boldsymbol{I}_{M}\right)$, clearly $\widetilde{N}:=$ $\boldsymbol{N}^{H} \boldsymbol{N} \sim \chi_{2 M}^{2}$ with probability density

$$
p_{\widetilde{N}}(\tilde{n})=\frac{M^{M}}{\sigma_{N}^{2 M} \Gamma(M)} \tilde{n}^{M-1} \mathrm{e}^{-\frac{M}{\sigma_{N}^{2}} \tilde{n}} \mathbb{1}_{[0, \infty)}(\tilde{n}),
$$

where $\Gamma(z), \operatorname{Re}\{z\}>0$, denotes the Gamma function. If we define $\breve{N}:=\frac{1}{\alpha} \widetilde{N}, \alpha>0$, the probability density changes to $p_{\breve{N}}(\breve{n})=\alpha p_{\widetilde{N}}(\alpha \tilde{n})$ and $\mathbb{E}\left\{\psi\left(\frac{1}{\alpha} \tilde{N}\right)\right\}$ can be written as

$$
\begin{aligned}
\mathbb{E}\{\psi(\breve{N})\} & =\int_{0}^{\infty} \psi(\breve{N}) p_{\breve{N}}(\breve{n}) d \breve{n} \\
& =\frac{(\alpha M)^{M}}{\sigma_{N}^{2 M} \Gamma(M)} \int_{0}^{\infty} \tilde{n}^{M-1} \mathrm{e}^{-\left(\frac{\alpha M K-\sigma_{N}^{2} \log _{\mathrm{e}}(a)}{\sigma_{N}^{2} K}\right) \tilde{n}} d \tilde{n}
\end{aligned}
$$

Note that

$$
\Gamma(z)=\int_{0}^{\infty} x^{z-1} \mathrm{e}^{-x} d x=k^{z} \int_{0}^{\infty} x^{z-1} \mathrm{e}^{-k x} d x
$$

for $\operatorname{Re}\{z\}, \operatorname{Re}\{k\}>0$ [6], so that with the presupposition $\sigma_{N}^{2} \log _{\mathrm{e}}(a)<\alpha K M$, a comparison of (18) with the righthand side of (19) yields the result.

(ii) Since $\left(\frac{\alpha K M}{\alpha K M-\sigma_{N}^{2} \log _{\mathrm{e}}(a)}\right)^{M}=\left(1-\frac{\sigma_{N}^{2} \log _{\mathrm{e}}(a)}{\alpha K M}\right)^{-M}$, the result follows with the fact that $\lim _{n \rightarrow \infty}\left(1-\frac{x}{n}\right)^{n}=\frac{1}{\mathrm{e}^{x}}, x \in \mathbb{R}$.

\section{APPENDIX B}

\section{PROOF OF LEMMA 2}

Proof: From [4] we conclude that for arbitrary but fixed $\underset{\widetilde{\Delta}}{\mathrm{\Delta}}<\infty$, compact $\mathcal{X}$, and $M$ large enough, the error-sum $\widetilde{\Delta} \mid \boldsymbol{x}$ (cf. (9)), conditioned on $\boldsymbol{X}=\boldsymbol{x}$, is approximately $\mathcal{N}_{\mathbb{R}}\left(\sigma_{N}^{2}, \alpha^{2} \sigma_{\breve{\Delta} \mid \boldsymbol{x}}^{2}\right)$ distributed. Thus, for $M$ large enough, $\widetilde{\Delta} \mid \boldsymbol{x}$ has approximately the distribution function $\widetilde{P}_{\widetilde{\Delta} \mid \boldsymbol{x}}(\tilde{\delta} \mid \boldsymbol{x})=$ $\frac{1}{2}+\frac{1}{2} \operatorname{erf}\left(\frac{\tilde{\delta} \mid \boldsymbol{x}-\sigma_{N}^{2}}{\sqrt{2} \alpha \sigma_{\breve{\Delta} \mid \boldsymbol{x}}}\right)$, i.e., $P_{\widetilde{\Delta} \mid \boldsymbol{x}}(\tilde{\delta} \mid \boldsymbol{x}) \approx \widetilde{P}_{\widetilde{\Delta} \mid \boldsymbol{x}}(\tilde{\delta} \mid \boldsymbol{x})$. Since $\Xi \mid \boldsymbol{x}=\psi\left(\frac{1}{\alpha} \widetilde{\Delta} \mid \boldsymbol{x}\right)=a^{\frac{1}{\alpha K} \widetilde{\Delta} \mid \boldsymbol{x}}, K, \alpha>0, a>1$, and $\psi$ a strictly monotonic increasing function, $P_{\Xi \mid \boldsymbol{x}}(\xi \mid \boldsymbol{x})=\mathbb{P}(\Xi \leq \xi \mid \boldsymbol{x})=$
$\mathbb{P}\left(\widetilde{\Delta} \leq \alpha K \log _{a}(\xi) \mid \boldsymbol{x}\right)=P_{\widetilde{\Delta} \mid \boldsymbol{x}}\left(\alpha K \log _{a}(\xi) \mid \boldsymbol{x}\right)$, from which with $M$ large enough $P_{\Xi \mid \boldsymbol{x}}(\xi \mid \boldsymbol{x}) \approx \widetilde{P}_{\Xi \mid \boldsymbol{x}}(\xi \mid \boldsymbol{x})$ with

$$
\widetilde{P}_{\Xi \mid \boldsymbol{x}}(\xi \mid \boldsymbol{x})=\frac{1}{2}\left(1+\operatorname{erf}\left(\frac{\log _{\mathrm{e}}(\xi \mid \boldsymbol{x})-\frac{\sigma_{N}^{2} \log _{\mathrm{e}}(a)}{\alpha K}}{\sqrt{2} \frac{\log _{\mathrm{e}}(a) \sigma_{\breve{\Delta} \mid x}}{K}}\right)\right),
$$

$\xi \mid \boldsymbol{x} \in \mathbb{R}_{++}$, follows. Note that (20) describes the distribution function of a log-normal distributed random variable with parameters $\frac{\sigma_{N}^{2} \log _{\mathrm{e}}(a)}{\alpha K}=: \mu_{\Xi}$ and $\frac{\left(\log _{\mathrm{e}}(a)\right)^{2} \sigma_{\Delta \mid x}^{2}}{K^{2}}=: \sigma_{\Xi \mid x}^{2}$, such that approximately $\Xi \mid x \sim \mathcal{L} \mathcal{N}\left(\mu_{\Xi}, \sigma_{\Xi \mid x}^{2}\right)$ holds.

\section{APPENDIX C}

\section{ProOF OF PROPOSITION 1}

Proof: Since we know that the distribution function of a log-normal distributed random variable $X$, i.e., $X \sim$ $\mathcal{L N}\left(\mu, \sigma^{2}\right)$, has the form $P_{X}(x)=\mathbb{P}(X \leq x)=\frac{1}{2}+$ $\frac{1}{2} \operatorname{erf}\left(\frac{\log _{\mathrm{e}}(x)-\mu}{\sqrt{2 \sigma^{2}}}\right)$, in addition $\mathbb{P}(X>x)=1-P_{X}(x)=$ $\frac{1}{2} \operatorname{erfc}\left(\frac{\log _{\mathrm{e}}(x)-\mu}{\sqrt{2 \sigma^{2}}}\right)$ holds. Thus, if we remind that $\Xi \mid x \sim$ $\mathcal{L} \mathcal{N}\left(\mu_{\Xi \mid \boldsymbol{x}}, \sigma_{\Xi \mid \boldsymbol{x}}^{2}\right)$ (cf. Lemma 2) and $\gamma>0$, follows from (12) $\mathbb{P}(E \leq e \mid \boldsymbol{x})=\mathbb{P}(\Xi \leq \gamma+\gamma e \mid \boldsymbol{x})=P_{\Xi \mid \boldsymbol{x}}(\gamma+\gamma e \mid \boldsymbol{x})$, $e \in \mathbb{R}$. Then, for $\epsilon>0, \mathbb{P}(|E|<\epsilon \mid \boldsymbol{x})=\mathbb{P}(-\epsilon<$ $E<\epsilon \mid \boldsymbol{x})=\left[P_{E \mid \boldsymbol{x}}(\epsilon \mid \boldsymbol{x})-P_{E \mid \boldsymbol{x}}(-\epsilon \mid \boldsymbol{x})\right] \mathbb{1}_{(0, \infty)}(\epsilon \mid \boldsymbol{x})=$ $P_{\Xi \mid \boldsymbol{x}}(\gamma+\gamma \epsilon \mid \boldsymbol{x}) \mathbb{1}_{(0, \infty)}(\epsilon \mid \boldsymbol{x})-P_{\Xi \mid \boldsymbol{x}}(\gamma-\gamma \epsilon \mid \boldsymbol{x}) \mathbb{1}_{(0,1)}(\epsilon \mid \boldsymbol{x})$. Taking the approximation (20) into account we get $1-\mathbb{P}(|E|<$ $\epsilon \mid \boldsymbol{x})=\mathbb{P}(|E| \geq \epsilon \mid \boldsymbol{x}) \approx \widetilde{\mathbb{P}}(|E| \geq \epsilon \mid \boldsymbol{x})=1+\widetilde{P}_{\Xi \mid \boldsymbol{x}}(\gamma-$ $\gamma \epsilon \mid \boldsymbol{x}) \mathbb{1}_{(0,1)}(\epsilon \mid \boldsymbol{x})-\widetilde{P}_{\Xi \mid \boldsymbol{x}}(\gamma+\gamma \epsilon \mid \boldsymbol{x}) \mathbb{1}_{(0, \infty)}(\epsilon \mid \boldsymbol{x})$, i.e.,

$\widetilde{\mathbb{P}}(|E| \geq \epsilon \mid \boldsymbol{x})=\left\{\begin{array}{cl}1+\widetilde{P}_{\Xi \mid \boldsymbol{x}}(\gamma-\gamma \epsilon \mid \boldsymbol{x})- & \widetilde{P}_{\Xi \mid \boldsymbol{x}}(\gamma+\gamma \epsilon \mid \boldsymbol{x}), \\ & 0<\epsilon<1 \\ 1-\widetilde{P}_{\Xi \mid \boldsymbol{x}}(\gamma+\gamma \epsilon \mid \boldsymbol{x}), & 1 \leq \epsilon<\infty\end{array}\right.$.

So this with (20) and $\operatorname{erfc}(x)=1-\operatorname{erf}(x), x \in \mathbb{R}$, proves the result.

\section{REFERENCES}

[1] A. Giridhar and P. R. Kumar, "Computing and communicating functions over sensor networks," IEEE J. Sel. Areas Commun., vol. 23, no. 4, pp. 755-764, Apr. 2005.

[2] - "Toward a theory of in-network computation in wireless sensor networks," IEEE Commun. Mag., vol. 44, no. 4, pp. 98-107, Apr. 2006.

[3] B. Nazer and M. Gastpar, "Computation over multiple-access channels," IEEE Trans. Inf. Theory, vol. 53, no. 10, pp. 3498-3516, Oct. 2007.

[4] M. Goldenbaum, S. Stańczak, and M. Kaliszan, "On function computation via wireless sensor multiple-access channels," in Proc. IEEE Wireless Communications \& Networking Conference (WCNC), Budapest, Hungary, Apr. 2009.

[5] STMicroelectronics, "Ultra-low current $2.4 \mathrm{~V}$ precision analog temperature sensor," STLM20 datasheet, Sep. 2009.

[6] M. Abramowitz and I. A. Stegun, Handbook of Mathematical Functions with Formulas, Graphs, and Mathematical Tables, ninth dover printing ed. New York: Dover, 1964. 